\title{
Analysis of the Rehabilitation Effect of Modern Rehabilitation Concept Combined with Acupuncture and Massage on Infantile Cerebral Palsy
}

\author{
Xiaosu Jie ${ }^{1, a}$, Haijun Shan ${ }^{1, b}$, Yujin Hou ${ }^{1, c}$, Hua Shi ${ }^{1, \text { d }}$, Yuanjun Lou ${ }^{1, \text { e }}$, Yingying Zhang ${ }^{1, f}$, Xin Guo ${ }^{1, \mathrm{~g}}$ \\ ${ }^{1}$ Henan province hospital of Traditional Chinese Medicine, Zhengzhou, Henan, 450002
}

\begin{abstract}
Objective: To explore the effect of combining modern rehabilitation concepts with acupuncture and massage therapy on the rehabilitation of children with cerebral palsy. Methods: Research work was carried out in our hospital. The work was carried out from September 2018 to September 2019. A total of 100 patients were selected, all of whom were children with cerebral palsy. The 100 patients with cerebral palsy were divided into two groups: the control group who was given modern rehabilitation concept intervention, and the intervention group who was given modern rehabilitation concept combined with acupuncture and massage. The recovery condition of the two groups of cerebral palsy children was compared and analyzed. Results: There were 5 patients in the treatment group who were ineffective, the effective rate was $90.00 \%$, and 11 people in the control group were ineffective, the effective rate was $78.00 \%$ which has existential meaning. The incidence of sequelae of the children in the experimental group was $14.00 \%$, and the incidence of sequelae of the children in the control group was $32.00 \%$. In contrast, the incidence of the experimental group was low and the effect was good. The difference in data was significant $(\mathrm{P}<0.05)$. Conclusion: The combination of modern rehabilitation concepts and TCM acupuncture and massage intervention methods in children with cerebral palsy has a significant effect, which can improve the child 's adverse conditions, promote the child 's recovery, and improve the child 's motor function, it can be promoted.
\end{abstract}

\section{1 introduction}

Cerebral palsy is a common clinical disease and belongs to a disease of the central nervous system. The main cause of the morbidity of the child is that the brain of the child is injured, or the child has developmental defects, which leads to the occurrence of cerebral palsy. It will be accompanied by movement disorders, intellectual disabilities and language disorders, which have a greater impact on the physical and mental health of the children. Judging from the current clinical development, children with cerebral palsy mostly use traditional Chinese medicine, sports, drugs and comprehensive rehabilitation and other treatment methods [1]. On the basis of this research, while applying the modern rehabilitation concept, the children were given acupuncture and massage therapy to explore the clinical intervention effects. The relevant analysis and reports are as follows.

\section{Patient data analysis and method research}

\subsection{General information}

The research work was carried out in our hospital from September 2018 to September 2019. A total of 100 patients were selected, all of whom were children with cerebral palsy. The 100 patients with cerebral palsy were divided into two groups, the control group who was given modern rehabilitation concept intervention, and the intervention group who was given modern rehabilitation concept combined with acupuncture and massage. The number of children in each group is 50 .

\subsection{Research methods}

The control group used intervention treatment of modern rehabilitation concepts, which mainly included posture, language, exercise ability and audio-visual. Holding material training, giving the child purposeful in-situ exercise, upper and lower limb functional exercises, etc., training once a day, resting one day after 6 days of continuous training, for a total of 3 months.

The children in the experimental group used modern rehabilitation concepts combined with traditional Chinese medicine acupuncture and massage. The modern rehabilitation concept intervention method was the same as the control group. Traditional Chinese medicine massage: In the specific implementation process, it is necessary to start from the meridian of the child, and give 
the child intervention such as pushing, holding and pinching. For children with spastic cerebral palsy, gentle techniques need to be applied to promote the child's physical and mental relaxation, for children with cerebral palsy with relatively low muscular tension, it is necessary to give the children plucking tendons and give them acupressure treatment, which mainly includes Fengchi, Mingmen, Quchi, Baihui, Huantiao and Ganyu and other points, in the process of pressing, it is necessary to ensure that the hand of the attending physician maintains close contact with the skin of the patient, avoids the separation between the fingers and the meridian points, and performs strict operations according to the principle of first-come-first-served basis. Acupuncture treatment: it is necessary to apply a 1.5-inch milli-needle at a 15-degree angle and pierce the milli-needle under the cap-shaped aponeurosis of the child, needle retention treatment is required when the needle feels stuck and there is no feeling of astringency, last for 30-45 minutes, at the same time select the intelligence zone, language two and three zones, sports zone and foot movement zone as the main needle insertion zone. In the process of applying needles, it is necessary to ensure stability, accuracy, and speed. For the children with deficiency, the supplementary method needs to be applied. The empirical children need to use the vent method. The children with deficiency and empirical evidence are not required to use the method of selective balance to relieve the laxative, after getting the Qi, leave the needle for 30 minutes. The main intervention points are Zusanli, Hegu, Baihui and Acupoints such as Sishencong.

\subsection{Observation indicators}

The treatment effect mainly includes three indicators of marked effect, effective and ineffective, among them, marked effect means that after receiving treatment, the child's muscle tone is improved, abnormal posture is controlled, and intelligence, language and developmental ability are improved, effective means that after receiving treatment, the child's abnormal posture and muscle tone are improved, and the language and intellectual response are improved compared with before treatment, ineffective, after receiving treatment, the child did not have any obvious improvement, and even appeared to be worse. Total effective rate $=$ significant rate + effective rate [2].

\subsection{Statistical methods}

Using the statistical software SPSS20.0 as a tool, performing statistical analysis on the data presented in this study, verifying the $t$ value of the comparison result of the measurement data $( \pm \mathrm{s})$, verifying the 2 value of the result of the count data $(\mathrm{n}, \%)$ when the result shows $\mathrm{P}<0.05$, it means that the difference between the groups has statistical analysis value [3]

\section{Results}

\subsection{Effective treatment}

There were 5 patients in the experimental group who were ineffective, the effective rate was $90.00 \%, 11$ ineffective in the control group, and the effective rate was $78.00 \%$. The effective rate in the experimental group was higher, and the data differences between the groups were significant

Table 1 Comparison of treatment efficiency between two groups of patients [n (\%)]

\begin{tabular}{ccccc}
\hline Group & $\begin{array}{c}\text { Marked } \\
\text { Effective }\end{array}$ & Effective & Ineffective & $\begin{array}{c}\text { Effective } \\
\text { Rate }\end{array}$ \\
\hline $\begin{array}{c}\text { Experimental } \\
\text { Group }\end{array}$ & $33(66.00)$ & $12(24.00)$ & $5(10.00)$ & $45(90.00)$ \\
$(\mathrm{n}=50)$ & & & & \\
Control & $20(40.00)$ & $19(38.00)$ & $11(22.00)$ & $39(78.00)$ \\
Group & & & & \\
$(\mathrm{n}=50)$ & & & & 12.354 \\
$\mathrm{X} 2$ & & & $<0.05$ \\
$\mathrm{P}$ & & & \\
\hline
\end{tabular}

\subsection{Comparison of the incidence of sequelae}

The incidence of sequelae of the children in the experimental group was $14.00 \%$, and the incidence of sequelae of the children in the control group was $32.00 \%$. In contrast, the incidence of the experimental group was lower and the effect was good. The data difference is significant.

Table 2 Comparison of the incidence of sequelae between two groups of children [n (\%)]

\begin{tabular}{|c|c|c|c|c|c|}
\hline Group & $\begin{array}{c}\text { Mental } \\
\text { retardation }\end{array}$ & Paralysis & $\begin{array}{l}\text { Language } \\
\text { disability }\end{array}$ & $\begin{array}{c}\text { Tooth } \\
\text { development }\end{array}$ & Incidence \\
\hline $\begin{array}{r}\text { Experimental } \\
\text { Group }(n=50)\end{array}$ & $2(4.00)$ & $1(2.00)$ & $2(4.00)$ & $2(4.00)$ & $7(14.00)$ \\
\hline $\begin{array}{l}\text { Control Group } \\
\quad(\mathrm{n}=50)\end{array}$ & $4(8.00)$ & $3(6.00)$ & $5(10.00)$ & $4(8.00)$ & $16(32.00)$ \\
\hline $\mathrm{X} 2$ & & & & & 9.225 \\
\hline $\mathrm{P}$ & & & & & $<0.05$ \\
\hline
\end{tabular}

\section{Discussion}

Pediatric cerebral palsy mainly refers to the brain damage problems that occur in children when they are not born or within one month of birth. Children often have problems with motor dysfunction and limb disorders, which lead to varying degrees of intelligence Defects, impaired motor perception and abnormal behavior [4]. Cerebral palsy is a common clinical disease and belongs to a disease of the central nervous system. The main cause of the morbidity of the child is that the brain of the child is injured, or the child has developmental defects, which leads to the occurrence of cerebral palsy. It will be accompanied by movement 
disorders, intellectual disabilities and language disorders, which have a greater impact on the physical and mental health of the children. Judging from the current clinical development, children with cerebral palsy mostly use traditional Chinese medicine, sports, drugs and comprehensive rehabilitation methods [5]. However, the effect of simple treatment is limited, and it is necessary to promote the comprehensive application of multiple methods in clinical practice.

Modern rehabilitation concept treatment refers to the rehabilitation training given to children based on the characteristics of the children's brain lesions and the movement disorders caused, starting from the theory of central nerve plasticity, and applying physical therapy and sports therapy, In the process of giving inhibitory tactics to the child, the abnormal posture and movement of the child are suppressed, thereby promoting the reflective movement of the child's body, and on this basis, bringing a negative effect on the physical induction of the child, It impedes the reflex pathway and plays an active role in promoting the normal function recovery of children [6]. However, the application effect of the simple modern rehabilitation concept is not significant enough, and it is necessary to use the Chinese medicine massage method in combination. Starting from the category of traditional Chinese medicine, children with cerebral palsy belong to the category of five late, five soft, etc. The disease of the child is basically in the brain. In the case of stagnation of the veins and the emptying of the brain marrow, it will lead to the occurrence of cerebral palsy in children. When the acupuncture method is applied, the brain of the child can be overflowed and the mind can be regulated. In the process of acupuncture and massage at the relevant points, it can cause a stimulating effect on the diseased area of the child, enhance its reflexivity, and affect the brain The cortex causes irritation, promotes blood flow velocity, restores the metabolic function of its brain cells, or strengthens its activated compensatory cells. At the same time, it can achieve the local or overall qi and blood condition and improve the body of the child. The balance of yin and yang can enhance the resistance of children, and achieve the effect of promoting blood circulation and removing blood stasis. The acupuncture method of traditional Chinese medicine is to improve the meridian airflow of the child and stimulate the acupuncture points to achieve the improvement of the central nervous system function of the child. The clinical effect is significant, the irritation is less, and the clinical tolerance of the child is stronger, it can promote the efficiency of clinical treatment, reduce the incidence of sequelae in children, and improve the prognostic effect of children with cerebral palsy [7].

The results of this study showed that: 5 people in the experimental group did not respond to treatment, the effective rate was $90.00 \%, 11$ people in the control group did not respond to the treatment, and the treatment effective rate was $78.00 \%$, The experimental group has a higher effective rate, the data difference between the groups is significant. The incidence of sequelae of the children in the experimental group was $14.00 \%$, and the incidence of sequelae of the children in the control group was $32.00 \%$. In contrast, the incidence of the experimental group was low and the effect was good. The difference in data was significant $(\mathrm{P}<0.05)$. The results show that the combination of modern rehabilitation concepts and traditional Chinese medicine acupuncture and massage can improve the clinical symptoms of children and promote their recovery.

In summary, the combination of modern rehabilitation concepts and TCM acupuncture and massage intervention methods in pediatric cerebral palsy has a significant effect, which can improve the child's adverse conditions, promote the child's recovery, improve the child's motor function, and have clinical development positive meaning, it can be promoted and used.

\section{References}

1. Wu Tao, Zhang Xia. Observation of the rehabilitation effect of modern rehabilitation concept combined with acupuncture and massage on infantile cerebral palsy [J]. Sichuan Journal of Traditional Chinese Medicine, 2016, 34 (03): 193-195.

2. Huang Fei. Effect of acupuncture and massage combined with rehabilitation measures on prognosis of cerebral palsy function recovery in children [J]. World Journal of Integrated Traditional Chinese and Western Medicine, 2019, 14 (04): 557-560.

3. Li Jianfeng. Observation on the rehabilitation effect of modern rehabilitation concept combined with acupuncture and massage on infantile cerebral palsy [J]. Famous Doctor, 2019 (07): 75.

4. Zhang Hongwei, Shao Yan. Application analysis of rehabilitation nursing plus acupuncture and massage in children with cerebral palsy [J]. China Continuing Medical Education, 2019, 11 (18): 136-138.

5. Zhao Surui. Analysis of the rehabilitation effect of modern rehabilitation concept combined with acupuncture and massage on children with cerebral palsy [J]. Chinese Journal of Integrated Traditional and Western Medicine Cardiovascular Disease, 2018, 6 (14): 135-136.

6. Xie Fang, Li Ying. Observation on the effect of acupuncture and massage on children with cerebral palsy [J]. Shaanxi Journal of Traditional Chinese Medicine, 2017,38 (01): 114-115.

7. Zhang Hongbo. The effect of comprehensive rehabilitation on the intelligent structure of children with cerebral palsy [D]. Jilin University, 2011. 